\title{
Cloud Storage Comparative Analysis Amazon Simple Storage vs. Microsoft Azure Blob Storage
}

\author{
Zouheir Daher and Hassan Hajjdiab
}

\begin{abstract}
A cloud computing service that allows data to be stored, retrieved and edited from a remote cloud storage server via Internet is called Cloud Storage Service . It delivers redundant, scalable and flexible storage capacity through Web Services Application Program Interface, thin client applications and online interfaces. Users' demand on cloud based storage services has increased rapidly which has led to high competition between the Storage Service Providers (SSPs) This resulted in cheaper price per GB, better service and enhanced Quality of Experience (QoE). Despite the high public interest in cloud storage services, users are still having serious concerns about selecting the right storage service and on what basis their selection should depend based on their storage requirements. This paper provides a detailed comparative analysis between two major popular cloud storage services: (1) Amazon Simple Storage Service (Amazon S3) and (2) Microsoft Azure Blob Storage. It first describes the storage classes /tires for each one and then presents analytical comparison for their Granularity, Service Availability, Pricing, Scalability, Regional Availability, Security, Changing Access Tier and Performance. By comparing two benchmarks storage services, I aim to provide users guidelines for what and how to compare several storage services to select the right one that suits their special requirements and demands.
\end{abstract}

Index Terms-Acess tier, granularity, performance, pricing, security, scalability, storage.

\section{INTRODUCTION}

More and more people are attracted by cloud storage services that allow them to synchronize files with servers in the cloud and among different devices. It facilitates automatic file sharing and synchronization among different users. Recently the cloud storage market has grown dramatically, and new players have emerged. High competition between companies resulted in cheaper price per GB, better service and enhanced QoE. Selecting the storage service that suits the users' specific needs has become a significant task.

This paper provides the results of my research for identifying and comparing a wide variety of factors of two benchmark cloud storage services Amazon S3 and Microsoft Azure Blob Storage. I have documented the results as follows:

- Section II defines the Amazon S3 and Azure Blob Storage services and explains in details the storage

Manuscript received November 10, 2017; revised January 18, 2018. The authors are with Abu Dhabi University College of Engineering, Abu Dhabi University, Abu Dhabi, United Arab Emirates (e-mail: 1063857@students.adu.ac.ae, Hassan.hajjdiab@adu.ac.ae). classes offered by each service with their features and usage purposes. It helps users to understand the storage concept so they can be able to select the right class based on their requirements.

- Section III to Section X: each section compares one of the following factors in each storage service: Granularity, Service Availability, Scalability, Pricing, Changing Access Tier Security, Regional Availability, and Performance.

- Section XI provides the conclusions of the comparison mentioned in the previous sections.

\section{StORAGe Classes}

\section{A. Amazon $\mathrm{S3}$}

Amazon S3 has a set of storage classes to suit different use cases. These include Standard for data that are frequently accessed and is called general-purpose storage, Standard for data that are infrequently accessed and longlived and is called Standard- Infrequent access, and Amazon Glacier for archive. Policies are configured to manage data throughout its lifecycle. When a policy is set, data migration to the most appropriate storage class will be done automatically without any application changes.

- Amazon S3 Standard provides high durability, performance and availability object storage for data that are frequently accessed. Due to its high throughput and low latency, Standard is perfectly suited for cloud applications, dynamic websites, mobile applications, gaming applications, big data analytics and content distribution. Policies are configured, by lifecycle management, for objects migration to appropriate storage class.

- Amazon S3 Standard - Infrequent Access (Standard IA) is a storage for less frequently accessed data but requires fast access when requested. Standard - IA provides high throughput, durability, and low latency with a low price and fee per GB storage and retrieval. Due to its high performance and low cost, Standard - IA is perfectly suited for disaster recovery, long-term storage and backups. Lifecycle policies can be used for automatic transferring objects, without application changes, between storage classes.

- Amazon Glacier is an extremely low cost, durable and secure storage service for archiving data. It provides a reliable storage for any amount of data with a cheaper or competitive cost with on-premises solutions. Lifecycle policies can be used for automatic transferring objects, without application changes, between storage classes. To satisfy varying retrieval needs with keeping costs low, 
Amazon Glacier offers three archives access options that varies from a short period (few minutes) to long period (several hours).

\section{B. Microsoft Azure Blob Storage Tiers and Replications}

Blob Storage, also known as Object Storage, is where unstructured object data can be stored. A blob can be binary data or any text type. It can be a media file, document, or application installer. Azure Storage provides two storage tiers for Blob object storage [1]-[4] so that data can be stored with most cost-effectively depending on its usage.

- The Azure hot storage tier is for storing frequently accessed data. Data requires high durability, availability, and quick time to access.

- The Azure cool storage tier is for storing data that is long-lived and infrequently accessed. Data requires high durability and similar time-to-access and throughput as hot data, but it can tolerate slightly lower availability.

To ensure high availability and durability, the data in storage account are always replicated. Based on replication option, data are either copied to a second data center or within the same data center. By replication, data availability is preserved and data are protected against any hardware failure. Replicating data to a second data center saves and protects data against a disaster failure in the primary location. MS Azure storage account provides these replication options:

- Locally redundant storage (LRS): It keeps three data copies within a single data center in a single region. It protects data from normal hardware failures, but not from the failure of a single data center. LRS is offered at a discount.

- Zone-redundant storage (ZRS): It maintains three copies of data across two to three facilities, across two regions or within a single region. It is more durable than LRS. It confirms the data durability within a single region. Once ZRS is selected, it cannot be converted to any other replication type.

- Geo-redundant storage (GRS): It keeps six copies of data. three copies within the primary region and three copies in a secondary region, providing the highest level of durability. In case of a failure at the primary region, Azure Storage failover to the secondary region. It ensures that data are durable in two separate regions.

- Read-access geo-redundant storage (RA-GRS): It replicates data to a secondary geographic location, and provides read access to data in the secondary location. Data can be accessed either from the primary or the secondary location, if one location becomes unavailable. RA-GRS is the default replication option.

\section{GRANULARITY}

Both Azure Blob and Amazon S3 allow setting information at different high or low frequency access but with granularity variation [5], [6]. In Amazon S3, an object use either S3 Standard or IA

In Azure, Hot Blobs (HBS) and Cool Blobs (CBS) are the two options from where the storage account must select one to use. Costs may incur when changing access-frequency tier.

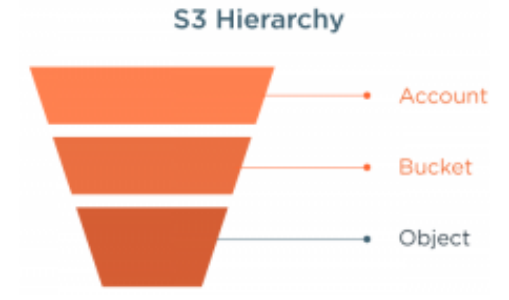

Fig. 1. S3 hierarchy.

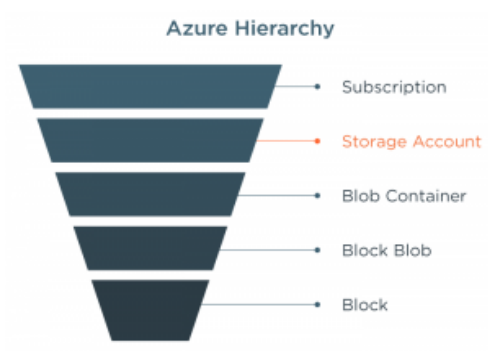

Fig. 2. Azure hierarchy.

\section{Availability SerVice LeVEl AgreEment (SLA)}

Both Amazon S3 and Azure provide a billing credit in SLA in case the service is down below an assigned monthly threshold. The availability SLA is less than the highfrequency-access tier so lower prices can be offered.

Both Azure and Amazon S3 provides a 25\% and $10 \%$ credit if availability is less than $98.0 \%$ and $99.0 \%$ respectively. Azure threshold is $99.9 \%$ for RA-GRS configurations. In Azure Hot Blob Storage and AMAZON S3 Standard, the availability credit thresholds are $99.9 \%$ and 99.0\% (and 99.99\%/99.0\% for RA-GRS) [6], [7].

TABLE I: AVAILABILITY SLA

\begin{tabular}{|c|c|c|c|}
\hline \multirow{2}{*}{$\begin{array}{c}\text { Service } \\
\text { Credit } \\
\%\end{array}$} & \multicolumn{3}{|c|}{ Storage Classes } \\
\hline & S3 Standard & S3 Standard IA & Azure Blob Storage \\
\hline $10 \%$ & $\begin{array}{l}\text { Equal to or } \\
\text { greater than } \\
99.0 \% \text { but } \\
\text { less than } \\
99.9 \%\end{array}$ & $\begin{array}{c}\text { Equal to or } \\
\text { greater than } \\
98.0 \% \text { but less } \\
\text { than } 99.0 \%\end{array}$ & $\begin{array}{c}\text { Less than99.0\% } \\
\text { read/write GRS, LRS, } \\
\text { ZRS, RA-GRS } \\
\text { Less than 99.9\% RA- } \\
\text { GRS-read }\end{array}$ \\
\hline $25 \%$ & $\begin{array}{ll}\text { Less } & \text { than } \\
99.0 \% & \end{array}$ & Less than $98.0 \%$ & Less than $98.0 \%$ \\
\hline
\end{tabular}

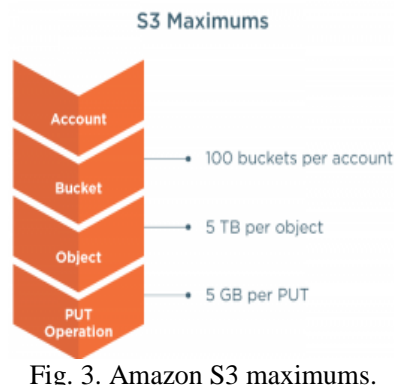

Fig. 3. Amazon S3 maximums.

\section{SCALABILITY}

An Azure subscription has a maximum of $100 \times 500$ TB accounts, with $4 \mathrm{MB}$ per block, approximately 195 GB per block blob, and with 500 TB per blob container (Note that number of accounts per subscription can be increased to more than 100 if requested). Amazon S3 has an upper limit of 100 buckets per account, 5GB per PUT operation and 5 TB per object. 
Transferring a large object is done by breaking it down into smaller chargeable transactions. Since CBS tiers are used for backup data, the number of transactions and the amount of data stored can be lowered by de-duplicating data before transferring it to cool storage tier.

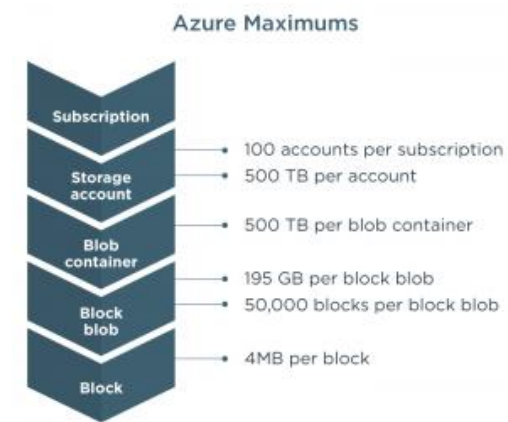

Fig. 4. Azure maximums.

TABLE II: SECURITY

\begin{tabular}{|c|c|c|}
\hline \multirow{2}{*}{ Data } & \multicolumn{2}{|c|}{ Storage Services } \\
\hline & Blob Storage (Azure) & Amazon S3 \\
\hline pre-flight & \multicolumn{2}{|c|}{ Encryption library- Client-side } \\
\hline in flight & \multicolumn{2}{|c|}{ Transport Layer Security -TLS } \\
\hline post-flight & Not available (in preview) & $\begin{array}{c}\text { Server-side Encryption } \\
\text { (AES-256) }\end{array}$ \\
\hline Key management & $\begin{array}{c}\text { Key Vault, or key provided by } \\
\text { customer }\end{array}$ & $\begin{array}{c}\text { S3-managed, Key } \\
\text { Management System, or } \\
\text { customer-provided }\end{array}$ \\
\hline
\end{tabular}

\section{SECURITY}

Security of Azure CBS and Amazon S3-IA is the same for Azure HBS and Amazon S3 Standard [8], [9]. The major difference between Azure and Amazon S3 security is the lack of server-side encryption for Azure blob storage. It is expected to be available on May 23, 2017.

Azure and Amazon S3 provide an encryption library client-side that able to use a cloud-managed (Amazon S3 KMS/Azure Key Vault) master key or a customer-managed master key. Encrypted data in-flight can be accessed, by both Azure and Amazon S3, by using TLS. For data-at-rest encryption, AES-256, Amazon S3 KMS or the customer key can be used by Server-Side Encryption.

\section{REGIONAL AVAILABILITY}

AMAZON S3 is available at all Amazon regions [6]:

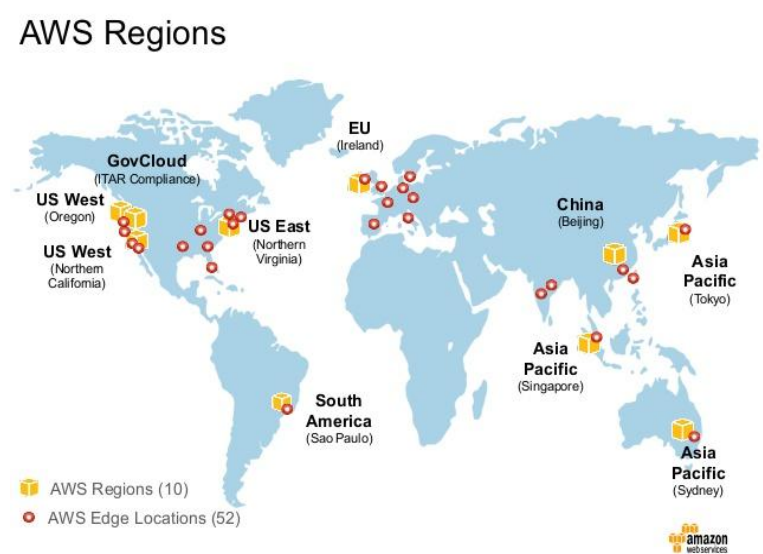

Fig. 5. Regions for Amazon S3.
Azure Blob Storage is available currently at: US (North Central, South Central, Central and East), Europe (North and West), Southeast Asia, Japan (East and West), India (Central, South and West):

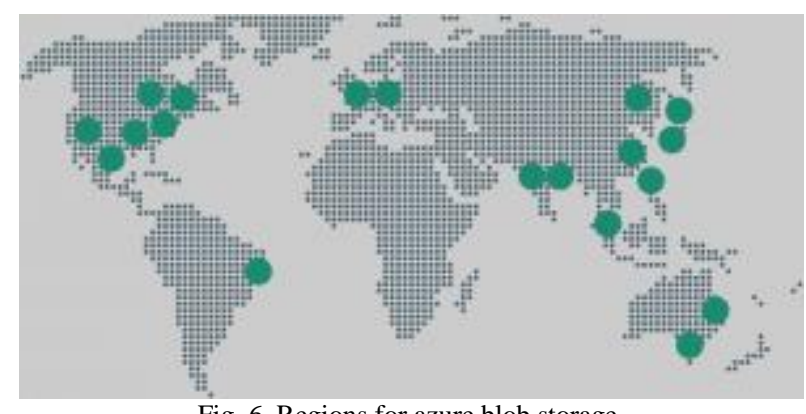

Fig. 6. Regions for azure blob storage.

\section{CHANGING ACCESS TIER}

Changing from a hot tier to the cool tier is possible in both Amazon S3 and Azure [6], [8].

In Amazon S3, Objects can be transferred from S3 Standard to S3-IA with a fee. To avoid further fee, don't move objects from their current tier until 30 days passed of their exiting in it."

In Amazon S3, Object Lifecycle Management is a tool that sets policies to migrate objects automatically among S3 storage classes based on a period or date. Polices setting occurs at the S3 bucket level.

In Azure, switching a hot blob storage account to cool is possible and free of charge. All objects within the account will be set as infrequently accessed. However, changing an existing general-purpose storage to a Cool Blob is impossible.

In Amazon S3, changing an object directly from S3Infrequent Access to a S3-Standard is not possible. There will be charges for S3-IA copying request and retrieving data. Changing from S3-IA to Glacier is possible.

In Azure, switching cool blob storage account to hot is possible with charges. All objects within the account will be set as frequently accessed and charges will be set as reading data charges in the storage account.

\section{PRICING}

Pricing is for the Azure East US 2 regions and Amazon S3 US East N. Virginia and as of this writing. The above table is a pricing sample:

Azure Cool Blob Storage and Amazon S3-IA Storage pricing is the addition of price for storing data, price for transferring data and price for requests.

Azure pricing depends on the selected redundancy which is GRS, LRS, RA-GRS or ZRS. Pricing for ZRS is the same as LRS.

Azure Cool Blob Storage LRS is cheaper than Amazon S3-IA for the cost of Data, but Amazon S3 is about 50\% cheaper than Azure Cool GRS and RA-GRS. In Amazon S3-IA, Deleting, overwriting or transferring objects to different storage class before 30 days of their existing incurs charges.

Data Transferred costs have two parts: Transfer in the cloud or transfer out of the cloud + Data retrieved or written 
to storage. Amazon S3 and Azure cost is \$0.01 per GB for data retrieval from storage and is free for data transfer into the cloud. Azure charges for the built-in GRD Transfer differently than transferring to another region.

TABLE III: PRICING SAMPLE

\begin{tabular}{|c|c|c|c|}
\hline & AMAZON S3-Infrequent Access & Cool Blob LRS-Azure & Cool Blob GRS \& RA-GRS Azure \\
\hline \multicolumn{4}{|c|}{ Data Storage } \\
\hline Amount of Data Stored & $\begin{array}{l}\$ 0.0125 / \text { GB / month less than } 30 \text { days: Pro- } \\
\text { rated storage charge. }\end{array}$ & $\$ 0.01 / \mathrm{GB} / \mathrm{month}$ & $\begin{array}{l}\text { RA-GRS: } \$ 0.025 / \text { GB / month } \\
\text { GRS: } \$ 0.02 \text { / GB / month }\end{array}$ \\
\hline \multicolumn{4}{|c|}{ Data Transfer } \\
\hline Data write & $\$ 0,0$ Free & $\$ 0.0025 / \mathrm{GB}$ & $\$ 0.005 / \mathrm{GB}$ \\
\hline Data retrieval & $\$ 0.01 / \mathrm{GB}$ & $\$ 0.01 / \mathrm{GB}$ & $\$ 0.01 / \mathrm{GB}$ \\
\hline Transfer IN to cloud & $\$ 0,0$ Free & $\$ 0,0$ Free & $\$ 0,0$ Free \\
\hline Transfer OUT to Internet & $\$ 0.09 / \mathrm{GB}$ & $\$ 0.087$ / GB & $\$ 0.087 / \mathrm{GB}$ \\
\hline Transfer TO another region & $\$ 0.020 / \mathrm{GB}$ & $\$ 0.087 / \mathrm{GB}$ & $\$ 0.087$ / GB \\
\hline Geo-Replication Data Transfer & Not Applicable & Not Applicable & $\$ 0.020 / \mathrm{GB}$ \\
\hline \multicolumn{4}{|c|}{ Requests } \\
\hline PUT & $\$ 0.10 / 10,000$ request & $\$ 0.10 / 10,000$ request & $\$ 0.20 / 10,000$ request \\
\hline POST (S3) / Create (Azure) & $\$ 0.10 / 10,000$ request & $\$ 0.10 / 10,000$ request & $\$ 0.20 / 10,000$ request \\
\hline COPY & $\$ 0.10 / 10,000$ request & $\$ 0.01 / 10,000$ request & $\$ 0.01 / 10,000$ request \\
\hline LIST & $\$ 0.01 / 10,000$ request & $\$ 0.10 / 10,000$ request & $\$ 0.20 / 10,000$ request \\
\hline GET & $\$ 0.01 / 10,000$ request & $\$ 0.01 / 10,000$ request & $\$ 0.01 / 10,000$ request \\
\hline DELETE & Free & Free & Free \\
\hline Other requests & $\$ 0.01 / 10,000$ request & $\$ 0.01 / 10,000$ request & $\$ 0.01 / 10,000$ request \\
\hline Lifecycle transition requests & $\$ 0.10 / 10,000$ request & Not Applicable & Not Applicable \\
\hline
\end{tabular}

Block Blob storage is used for streaming and storing pictures, documents, videos, backups, and other binary data or unstructured text. Table IV shows the pricing for dedicated Blob storage accounts with Hot and Cool access tiers.

The prices shown below are data storage monthly charges per GB. It varies based on the access tier and the redundancy option selected, as well as the amount of data stored.

TABLE IV: AZURE BLOB STORAGE PRICING

\begin{tabular}{|c|c|c|c|}
\hline \multirow{2}{*}{\multicolumn{2}{|c|}{ Storage Amount }} & \multicolumn{2}{|c|}{ Storage Prices (US Dollars) } \\
\hline & & HOT TIER & COOL TIER \\
\hline \multirow{3}{*}{$\begin{array}{l}\text { First } \\
\text { TB/month }\end{array}$} & \multirow{3}{*}{50} & LRS: $\quad \$ 0.0208$ & LRS: $\quad \$ 0.0152$ \\
\hline & & GRS: $\quad \$ 0.0458$ & GRS: $\quad \$ 0.0334$ \\
\hline & & RAGRS: $\$ 0.0478$ & RAGRS: $\$ 0.035$ \\
\hline \multirow{3}{*}{$\begin{array}{l}\text { Next } \\
\text { TB/month }\end{array}$} & \multirow{3}{*}{450} & LRS: $\quad \$ 0.02$ & LRS: $\quad \$ 0.0152$ \\
\hline & & $\$ 0.044$ & $\$ 0.0334$ \\
\hline & & RAGRS $\$ 0.0459$ & RAGRS: $\$ 0.035$ \\
\hline \multirow{3}{*}{$\begin{array}{l}\text { Over } \\
\text { TB/month }\end{array}$} & \multirow{3}{*}{500} & LRS: $\quad \$ 0.0192$ & LRS: $\quad \$ 0.0152$ \\
\hline & & $\$ 0.0422$ & $\$ 0.0334$ \\
\hline & & RAGRS: $\$ 0.044$ & RAGR : \\
\hline
\end{tabular}

Table V shows the Amazon S3 storage pricing [7], [10]:

\begin{tabular}{|c|c|c|c|}
\hline \multirow[b]{2}{*}{$\begin{array}{l}\text { Storage } \\
\text { Amount }\end{array}$} & \multicolumn{3}{|c|}{ Storage Prices (US Dollars) } \\
\hline & Standard Storage & \begin{tabular}{|c|} 
Standard - \\
Infrequent Access \\
Storage
\end{tabular} & Glacier Storage \\
\hline $\begin{array}{ll}\text { First } 50 & \text { TB } \\
\text { month } & \\
\end{array}$ & $\$ 0.026$ per GB & $\$ 0.019$ per GB & $\$ 0.005$ per GB \\
\hline $\begin{array}{l}\text { Next } 450 \text { TB } \\
\text { month }\end{array}$ & $\$ 0.025$ per GB & $\$ 0.019$ per GB & $\$ 0.005$ per GB \\
\hline $\begin{array}{l}\text { Over } 500 \mathrm{~TB} \\
\text { month }\end{array}$ & $\$ 0.024$ per GB & $\$ 0.019$ per GB & $\$ 0.005$ per GB \\
\hline
\end{tabular}

\section{PERformanCE}

Latency and Bandwidth for Cool Blob Storage and S3-IA are not significantly different than the higher-frequency tier. No performance targets have been published by Amazon for AMAZON S3 [1], [2], [6].

Below are some performance targets for storage published by Azure:

- 500 requests or $60 \mathrm{MB}$ per second or for single blob.

- 10 Gbps ingress, 30 Gbps egress per GRS account

- Request rate: 20,000 IOPS for a block size $1 \mathrm{~KB}$ per storage account

- 30 Gbps egress, 20 Gbps ingress per LRS account

\section{CONCLUSION}

Azure Cool Blob Storage and Amazon S3 Infrequent Access are suitable object storage tiers for backup and use cases where lot of data need to be stored but accessed infrequently with quick access when needed. They decrease the costs associated for storing objects. So, they are preferred reducing backup options for backing remote servers and cloud servers to object storage.

Azure requires the same tier for all storage account objects, whereas Amazon S3 allows setting the accessfrequency-tier for each object. So, it is wise to use Azure Blob Storage for creating a dedicated CBS account to backup data without using it for primary storage.

Azure Blob Storage and Amazon S3 Availability SLAs, are similar with an advantage of $0.9 \%$ for Azure RA-GRS read requests.

Scalability and Performance of Amazon S3-IA and Azure CBS match with S3 Standard and Azure HBS. This is suitable for small windows backup and quick restores as needed.

Both Azure Blob Storage and Amazon S3 provide key management and encryption for data in \& pre-flight but Azure is not offering post-flight encryption.

Azure Blob Storage is not available in all regions. For example, it is unavailable in Australia, and Singapore. Amazon S3 is available at all Amazon regions.

Reducing the frequency access tier of an Amazon S3 lifecycle management tool. Directly backing up to the S3IA tier is favorable for On-premise server backup customers, whereas for cloud server backup it is better to create a hot tier snapshot and then transfer it to a cool tier.

Deciding between using Amazon S3 or Azure Blob 
Storage is a case-by-case basis, but both decrease the cost of backup object storage. Users must take into consideration effective cost and right capabilities offered by public cloud service for use as a backup target [2], [6].

\section{REFERENCES}

[1] E. Bocchi, M. Mellia, and S. Sarni, "Cloud storage service benchmarking: Methodologies and experimentations," in Proc. 2014 IEEE 3rd International Conference on Cloud Networking (CloudNet), Luxembourg, 2014, pp. 395-400, vol. 1.

[2] T. Vijayakumar, Practical Azure Application Development: A Stepby-Step Approach to Build Feature-Rich Cloud-Ready Solutions, 1st ed. Apress, Berkely, CA, USA.2017.

[3] T. Madhuri and P. Sowjanya, "Microsoft Azure v/s Amazon AWS cloud services: A comparative study," International Journal of Innovative Research in Science, Engineering and Technology, vol. 5, issue 3, pp. 3904-3907, March 2016

[4] Introduction to Microsoft Azure Storage. (2017). [Online]. Available: https://docs.microsoft.com/en-us/azure/storage/storageintroduction\#introducing-the-azure-storage-services

[5] S. Samundiswary and N. M. Dongre, "Object storage architecture in cloud for unstructured data," in Proc. 2017 International Conference on Inventive Systems and Control (ICISC), Coimbatore, India, 2017, pp. 1-6.

[6] V. Persico, A. Montieri, and A. Pescapè, "On the Network Performance of Amazon S3 Cloud-Storage Service," in Proc. 2016 5th IEEE International Conference on Cloud Networking (Cloudnet), Pisa, 2016, pp. 113-118.

[1] Azure Storage Pricing. [Online]. Available: https://azure.microsoft.com/en-us/pricing/details/storage/blobs

[7] S. Krishnan, Programming Windows Azure: Programming the Microsoft Cloud. O'Reilly, 2010.

[8] Z. Hill, J. Li, M. Mao, A. Ruiz-Alvarez, and M. Humphrey, "Early observations on the performance of windows azure," in Proc. the 19th ACM International Symposium on High Performance Distributed Computing, 2010, pp. 367-376.

[9] Amazon S3 Pricing. [Online]. Available: https://aws.amazon.com/s3/pricing/

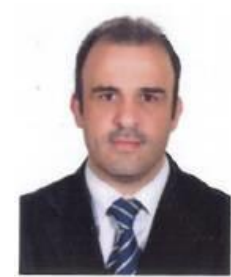

Zouheir Daher received his B. Sc. in computer science on Feb 1993 from the American University of Beirut, Lebanon, and soon got the M.SC in information technology from Abu Dhabi University, UAE.

$\mathrm{He}$ is currently working as an IT project manager at Abu Dhabi National Chemicals Company, UAE. He worked as a senior systems analyst at Abu Dhabi National Oil Company for 6 years. He served as an ICT teacher and trainer for 8 years in different schools and professional training centers.

Mr. Daher is a member in Project Management Professionals (PMP: PMI) as well as AUB Alumni Group

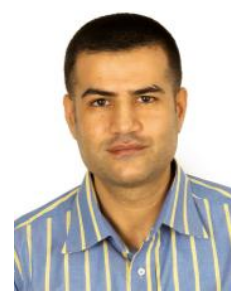

Hassan Hajjdiab received his B.Sc. in computer and communication engineering from the American University of Beirut, Lebanon and M.Sc. and Ph.D. degrees in computer science from the University of Ottawa, Canada.

$\mathrm{He}$ is an associate professor at Abu Dhabi University, United Arab Emirates and the Chair of the Computer Science and Information Technology department. His research interests are mobile computing, computer vision, image processing and remote sensing. Dr Hajjdiab is a member of the IEEE computer Society and has authored or co-authored many technical publications in refereed journals and conference. 